\title{
Composite Materials
}

\section{Editorial}

A noteworthy feature of the last two decades is the remarkable development of the type of new materials known as composites. They are encountered in almost every branch of industrial processing, from the most humble to the most advanced, and are taking over the place formerly occupied by conventional materials. The intrinsic physical limitations of the latter make them incapable of satisfying all the needs of modern technology. Plastics, for example, are affected by heat and lack mechanical resistance; metals are stronger but tend to be too heavy for aerospatial applications; ceramics have both low density and high thermal, chemical and mechanical resistance but are far too fragile. It was therefore natural to revise the idea of combining in a single substance materials with complementary characteristics, and this has led to the development of these new composites. Such materials are hence polyphasic solid in which two or more constituents are associated with a view to creating a substance possessing properties that none of the individual constituents possessed in isolation.

For thermomechanical applications, composites fall into three broad categories, depending on the nature of the matrix:

(i) Polymer-matrix composites (PMC), of which the best known and oldest are resins reinforced with glass fibres. The presence of the latter increases the mechanical resistance, the rigidity and the resistance to temperature variations vary considerably.

(ii) Metal-matrix composites (MMC). Reinforcement of a matrix consisting of a light metal (aluminium, titanium, magnesium...) with ceramic fibres (silicon carbide, alumina) creates a material, the strength, rigidity, resistance to fatigue and to high temperature of which are remarkable. Materials of this kind are nowadays indispensable in the armament, aerospace and automobile industries.

(iii) Ceramic-matrix composites (CMC). The matrix is a ceramic and a variety of types of reinforcement are in use: long or short fibres, particles, platelets, ...; their purpose is to take up as much elastic energy as possible whenever a fissure develops. Such materials are no longer more laboratory curiosities but are finding application in machine tools, armour and in the aerospace and car industries.

The complementary effect of the combination of matrix and reinforcement is occasionally paradoxical. Addition of ceramic particles or fibres to a ceramic can produce a material three times as tough; the sum of two weak materials is a much tougher one! This behaviour leads us to consider a third phase in these multiple materials; this phase, which is quasi-two-dimensional and is the sum of all the interfaces separating matrix and reinforcement, largely governs the final behaviour of the material.

In composites intended for thermomechanical applications, the degree of mechanical strength of these interfaces may render the material very rigid or, by participating in the dissipation of elastic energy under load, gives it greater resiliance. 
When we seek to understand or improve such materials, it is no longer sufficient to evaluate their properties in terms of grain size or the laws of mixtures. The damage and exchange mechanisms must be studied locally, close to the interfaces. This implies that the material must be examined and characterized on the scale of nanometres. The tool for such studies is clear: this is work for the electron microscopist.

Few research groups have ventured into this field, where the experimental difficulties are considerable: preparation of thin films from bulk specimens that are heterogeneous by definition; operation in low dose conditions, to avoid undesirable effects on certain ceramics and catastrophic damage to organic substances; interpretation of the contrast at interfaces where there may be abrupt changes in thickness; measurement of the light elements in these zones. There are a few examples of the problems to be overcome before a clear picture can emerge. Once they have been surmounted, however, considerable progress will be made in our understanding of the behaviour of the composite in question.

The chemical nature of composites, which often consists of light elements, and the dimensions of the zones to be examined, which are often of the same order of magnitude as the resolution of the experimental techniques employed, are such that the microscopist is driven to use his instrument at the limit of its capabilities. The development of better composites is thus intimately linked to improvement in the performance of analytical microscopy.

The object of the symposium on the study of composites, held in Toulouse in July 1990 during the 30th Annual Meeting of the SFME, was to bring together recent results obtained by groups in the zone of intersection between "composites" and "electron microscopy". The articles in this issue form a record of the papers presented during the meeting. The coverage cannot be exhaustive. For reasons of time and space, we had to be selective but we attempted to maintain a reasonable balance between the various types of composites and methods of study, always subject to the industrial restrictions on confidentiality which sometimes apply to these materials.

Very recent results concerning the following topics are included here:

- The use of conventional microscopy to study damage to interface at high temperature;

- Exploitation of high-resolution images to determine the structure at the atomic level of the different constituents of composites, such as carbon or silicon carbide fibres and the silicon carbide matrix;

- Application of the methods of X-ray analysis and EELS, with the associated displays, to study the mechanism of elemental diffusion towards the interfaces as a function of the conditions of preparation and treatment of the material.

In view of the diversity of the contributions, we felt it necessary to provide a general introduction on the fibres employed for reinforcement, which would impose some pattern on the individual papers. One of the principal specialists in this field consented to prepare such a survey.

The novelty and originality of the very concept of a composite material, the complexity of the structures within such substances and the understanding of the macroscopic properties raise problems of a fundamental and technological nature, which merit the attention of physicists. We trust that the results presented in these six articles will encourage research groups from both fields, electron microscopy and composite materials, to follow the example of the composites by reinforcing their collaboration.

\section{J. SEVELY CEMES - LOE/CNRS Toulouse}

\author{
A. THOREL \\ Ecole des Mines de Paris \\ Centre des matériaux \\ Evry
}

